Mendes, C. C., Couto, J. P., Ferreira, J. V. \& Mendes, E.

Causas dos desvios orçamentais desfavoráveis e os seus constructos: o caso dos projetos das empresas de construção portuguesas

\title{
CAUSAS DOS DESVIOS ORÇAMENTAIS DESFAVORÁVEIS E OS SEUS CONSTRUCTOS: O CASO DOS PROJETOS DAS EMPRESAS DE CONSTRUÇÃO PORTUGUESAS
}

\section{CAUSES OF UNFAVORABLE BUDGET DEVIATIONS AND THEIR CONSTRUCTS: THE CASE OF PORTUGUESE CONSTRUCTION COMPANIES 'PROJECTS}

\section{Caropul Capembute Mendes}

Instituto Superior de Engenharia de Coimbra

Guiné-Bissau

ORCID: 0000-0002-0499-3623

E-mail: caropul.mendes@isec.pt

\section{João Pedro Couto}

Escola de Engenharia da Universidade do Minho

Portugal

ORCID: 0000-0001-9607-0596

E-mail:.jpc@civil.uminho.pt

\section{José Vaz Ferreira}

Faculdade de Economia da Universidade de Coimbra

$$
\text { Portugal }
$$

ORCID: 0000-0002-7797-9486

E-mail: jvazfer@,fe.uc.pt

\section{Eulino Mendes}

Guiné-Bissau

Faculdade de Economia da Universidade de Coimbra

ORCID: 0000-0002-6589-6501

E-mail: limendes30@hotmail.com

\section{RESUMO}

As empresas de construção preocupam-se cada vez mais com novas orientações estratégicas e formas de gestão dos orçamentos dos projetos para melhorarem a sua competitividade e sustentabilidade. Nesta linha de pensamento, procurou-se analisar a relação dos constructos que determinam as causas dos desvios orçamentais desfavoráveis nos projetos das empresas de construção Portuguesas. Para o seu desenvolvimento foram adotados métodos e técnicas de investigação quantitativa. Os resultados apresentados no estudo mostram uma elevada correlação entre os cinco constructos de primeira ordem (ambiente interno, ambiente externo,

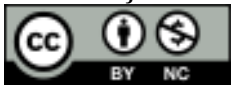


tempo/atraso, complexidade do projeto e financiamento do projeto), fundamentada pela revisão da literatura, justificam a compreensão relativa à identificação dos fatores que determinam as causas dos desvios orçamentais desfavoráveis nos projetos das empresas de construção Portuguesa. De salientar que, os objetivos traçados para este estudo foram alcançados ao disponibilizar os conhecimentos complementares nesta área de investigação, não apenas em termos científicos, mas também em termos práticos.

Palavras-chave: Causas dos desvios orçamentais desfavoráveis, Orçamento, Empresas de construção, Modelo de equação estrutural.

\section{ABSTRACT}

Construction companies are increasingly concerned with new strategic guidelines and ways of managing project budgets to improve their competitiveness and sustainability. In this line of thought, we sought to analyze the list of constructs that determine the causes of unfavorable budgetary deviations in the projects of Portuguese construction companies. For its development, quantitative research methods and techniques were adopted. The results presented in the study, show a high correlation between the five first order constructs (internal environment, external environment, time / delay, project complexity and project financing), based on the literature review, justify the understanding related to the identification of the factors that determine the causes of unfavorable budgetary deviations in the projects of Portuguese construction companies. It should be noted that the objectives set for this study were achieved by making complementary knowledge available in this area of research, not only in scientific terms, but also in practical terms.

Keywords: Causes of unfavorable budget deviations, Budget, Construction companies, Structural equation model.

\section{INTRODUÇÃO}

Desde os estudos de Welsch (1957) e de Anthony (1970), versando sobre planeamento e controlo dos lucros "Profit Planning and Control" e principio de contabilidade de gestão "Management Accounting Principles", que o orçamento tornou-se num tema que tem merecido atenção dos académicos e investigadores. Todavia, estes estudos contribuíram para disseminação do orçamento a nível dos projetos empresárias nomeadamente nos projetos das empresas de construção.

Os projetos e as atividades gerais das empresas de construção possuem uma enorme acuidade na economia nacional, particularmente no mercado de trabalho. Também são conhecidos as suas diversas atividades e áreas de negócios, tais como a construção civil, a engenharia civil, materiais de construção e entre outras atividades especializadas.

No entanto, quando se compara os projetos das empresas de construção Portuguesas com as outras empresas do mesmo ramo na Europa, verifica-se uma lacuna em termos de competitividade, devido ao reconhecimento de problemas como desvios orçamentais desfavoráveis, incumprimento de prazos, insuficiente qualidade do produto final, e também deficiências no que toca ao garante da segurança no trabalho (Couto, 2007).

A prevenção das lacunas em termos de competitividade possibilita revelar que o orçamento é uma ferramenta de planeamento estratégico, que proporciona aos gestores das empresas, em particular do sector da construção, informações úteis, quer para o 
acompanhamento da gestão corrente desenvolvida pela empresa, quer para a gestão dos projetos das empresas de construção (Coulmas \& Law, 2010). Apesar do orçamento estabelece uma mais-valia na gestão dos projetos, em particular nos projetos das empresas de construção, o mesmo é alvo de críticas frequentes.

Para Hansen et al. (2003) o orçamento encontra-se afastado da ideia de que possa constituir um sistema de controlo de gestão ótimo. Libby \& Lindsay (2007) salientam que as críticas sobre a insatisfação com os orçamentos têm aumentado nos últimos tempos. Estas críticas de insatisfação com o orçamento revelam que as empresas que operam sob condições de rápida mudança de mercado, não conseguem usufruir de medidas certas para o orçamento, devido à incerteza dos pressupostos a utilizar, assim como à morosidade no seu processo de elaboração. Apesar destas críticas de insatisfação e, com o desenvolvimento orçamental têm surgido varias técnicas a nível da sua execução, sendo ainda utilizados pela maioria das empresas a nível mundial (Ekholm e Wallin, 2000; Libby e Lindsay, 2010).

As previsões incluídas nos orçamentos, a curto, médio e longo prazo, são muito frequentemente afetadas por desvios orçamentais desfavoráveis devido às incertezas e condições dos mercados (Barki, Rivard, \& Talbot, 1993; Nidumolu, 1995). Contudo, os desvios orçamentais desfavoráveis estão destacados no fracasso de muitos projetos das empresas de construção (Mendes, 2019).

Todavia, depreende-se que é preciso e muito útil fazer um estudo aprofundado sobre as causas dos desvios orçamentais desfavoráveis aproveitando dados da tese de doutoramento intitulada "Determinantes dos Desvios Orçamentais e o seu Impacto na Performance das Empresas de Construção Portuguesas" (Mendes, 2019).

Para compreender a causas dos desvios orçamentais desfavoráveis nos projetos das empresas de construção, considera-se relevante mencionar os estudos que analisam as "derrapagens" de custos naquele tipo de projetos, dada a sua capacidade de compreender as diferenças entre as previsões e os custos reais, nos quais se constata que a maioria das empresas considera o excesso de custo como um fator crítico na prossecução dos objetivos dos projetos (Cantarelli, van Wee, Molin, \& Flyvbjerg, 2012; Lind \& Brunes, 2015; Odeck, 2004; Zhao, Wang, Mbachu, \& Liu, 2019; Zidane \& Andersen, 2018).

Sridarran et al. (2017) salientaram que o excesso de custos constitui uma das principais causas que determinam o fracasso nos projetos das empresas de construção. Estes autores afirmaram que, a dimensão do projeto e a complexidade do projeto são as principais causas que determinam o excesso de custo. Contudo deduz-se que, para evitar o excesso de custo ou desvios orçamentais desfavoráveis, a tendência é aumentar os lucros dos projetos das empresas de construção, caso seja possível identificar, corrigir, eliminar e controlar as suas causas (Cheng, 2014).

Refletindo em torno deste trabalho e sobre a respetiva investigação, depreende-se que é preciso sistematizar o conhecimento científico sobre esta temática e se possível introduzir novas abordagens com características inovadoras, acrescentado o valor a gestão dos projetos das empresas de construção. Portanto, justifica-se desenvolver um trabalho que procure perceber o distanciamento do orçamento previsto com o real, designado como desvios orçamentais desfavoráveis.

Todavia, ao considerar o interesse do estudo, o presente trabalho tem como "objetivo analisar a relação dos constructos que determinam as causas dos desvios orçamentais desfavoráveis nos projetos das empresas de construção Portuguesas". Para alcançar o objetivo pretendido, propôs-se inicialmente um modelo de investigação com os respetivos constructos nomeadamente, ambiente organizacional interno e externo do projeto, tempo e atraso do projeto, complexidade do projeto e financiamento do projeto. 
Em termos metodológicos, e tendo em conta a necessidade para avaliar o modelo proposto e testar as hipóteses formuladas, considera-se a utilização do modelo de equações estruturais (MEE) como a mais adequada para este estudo (Hair et al., 2014). A abordagem da equação estrutural tem sido explorada em muitos estudos para examinar as relações causais e testar as hipóteses nas várias áreas do ensino nomeadamente, na educação, na gestão e na economia (Xiong, Skitmore, \& Xia, 2015).

De salientar que este estudo pretende desenvolver os seguintes pontos: na segunda seção (ii), as variáveis explicativas das causas dos desvios orçamentais desfavoráveis são ilustradas conceitualmente à luz da literatura relacionada, e desenvolve-se as hipóteses do modelo de investigação. Na terceira seção (iii), a amostra, a recolha de dados e método de investigação são discutidas. Na quarta seção (iv), os resultados das análises são apresentados e descuidas, e na última seção (v), procurou-se discutir as conclusões bem como as contribuições, os aspetos que limitam o estudo e as sugestões para futuras investigações.

\section{REVISÃO DA LITERATURA}

Pretende-se nesta secção explorar a literatura especializada com o propósito de apresentar e analisar as principais dimensões do estudo (ambiente interno e externo da organização, tempo/atraso do projeto, complexidade do projeto e financiamento do projeto), também apresentar o modelo conceptual do estudo e as suas respetivas hipóteses.

\subsection{Ambiente interno e externo da organização do projeto como causas dos desvios orçamentais de favoráveis}

O ambiente organizacional tornou-se interessante no campo da investigação científica, devido às suas mudanças que compreendem as principais causas da difícil gestão dos objetivos da organização (Drucker, 2009).

O ambiente organizacional é considerado como a totalidade dos fatores físicos e sociais que são reconhecidos diretamente na tomada de decisão da organização (Duncan, 1972).

Para Mao, Zu \& Wang (2013), o ambiente organizacional é a soma de vários pontos dentro da organização que podem ser internos e externos capazes de ter uma certa influência nos desvios orçamentais desfavoráveis dos projetos das empresas de construção.

A capacidade de resposta dos orçamentos num ambiente dinâmico é agora questionada, como se comprova no estudo de (Lorain, 2010) relativo ao ambiente organizacional dinâmico nas empresas Espanholas. O mesmo estudo revelou que mais de $60 \%$ dos entrevistados considera as mudanças no ambiente organizacional como um fator difícil no estabelecimento de orçamento previsto. $\mathrm{O}$ autor salienta ainda que, com o ciclo de crise económica, o estabelecimento de previsões financeiras confiáveis compreende um esforço enorme.

Assim para compreender o esforço da previsão do orçamento confiável e das causas dos desvios orçamentais desfavoráveis, é imprescindível envolver os constructos das variáveis que definem os fatores interno e externo do ambiente daquele projeto de construção (Mendes, 2019).

Os fatores do ambiente interno que determinam os desvios orçamentais desfavoráveis nas empresas de construção são mensurados dentro dos limites da organização, através do espírito de equipa, do conflito entre unidades, da alteração frequente no sistema de gestão e da 
fraca liderança das equipas do trabalho (Duncan, 1972; Mao et al., 2013; Milliken, 1987; Worthington \& Britton, 2009). Na mesma linha de pensamento, o fator do ambiente externo são mensurados, de acordo com as medidas que se encontram fora dos limites da organização, nomeadamente condições climatéricas, alteração na estrutura do mercado, alterações imprevisíveis nos aspetos sociais, políticas e culturais no meio onde a empresa atua (Duncan, 1972; Hartmann, 2006; Mao et al., 2013; Worthington \& Britton, 2009).

Neste contexto, compreende-se a necessidade de procurar compreender as causas dos desvios orçamentais desfavoráveis, através do ambiente interno e externo da organização e a sua relação com o fator tempo/atraso, fator complexidade do projeto e fator financiamento do projeto das empresas de construção portuguesa.

\subsection{Fator tempo/atraso do projeto como causa dos desvios orçamentais desfavoráveis}

Com a dinâmica do mercado, percebe-se cada vez mais a existência dos fatores que contribuem para atrasos nos projetos das empresas de construção (Zidane \& Andersen, 2018). O fator tempo de concretização das atividades de um projeto de construção é sem dúvida um dos fatores que afeta a causa dos desvios orçamentais desfavoráveis. A variável "tempo de concretização de um projeto de construção", continua a não ter solução precisa, devido à sua enorme complexidade e interdependência com outras variáveis causadoras dos desvios orçamentais desfavoráveis (Couto, 2007).

Os atrasos ocorrem na maioria dos projetos de construção e a grandeza desses atrasos varia consideravelmente de projeto para projeto (Zidane \& Andersen, 2018). Todavia, é importante definir os fatores reais do tempo/atraso que explicam a variável tempo, para minimizar, mitigar e evitar atrasos em qualquer projeto de construção.

Os fatores que determinam os atrasos são cruciais dentro de um projeto de construção e é relevante que todas as organizações tenham conhecimento sobre esse assunto para que o projeto seja concluído dentro dos prazos estabelecidos (Wong \& Vimonsatit, 2012). Estes autores salientam ainda que, os atrasos provocam desvios orçamentais desfavoráveis, podendo vir a pôr em causa a viabilidade económica do projeto.

Contudo é importante definir as métricas do constructo tempo/atraso de modo a possibilitar a sua mensuração através de alterações dos projetos, do planeamento, do incumprimento dos detalhes do contrato, da escassez de materiais etc. (Arditi, Akan, \& Gurdamar, 1985; Cantarelli et al., 2012; Couto, 2007; Flyvbjerg, Skamris Holm, \& Buhl, 2004; Kaming, Olomolaiye, Holt, \& Harris, 1997).

De salientar que, para além do ambiente organizacional e do tempo de atraso, também é importante examinar outros fatores que causam desvios orçamentais desfavoráveis nos projetos das empresas da construção nomeadamente a complexidade do projeto e o método do financiamento dos projetos de construção.

\subsection{Fator complexidade do projeto como causa dos desvios orçamentais desfavoráveis}

A complexidade do projeto é compreendida como a caraterística de um projeto que dificulta a perceção da previsão, do controlo, da interdependência dos objetivos e da dependência entre cronogramas, mesmo quando são fornecidas informações razoavelmente completas sobre o sistema de execução do projeto (Brockmann \& Girmscheid, 2007; Remington, Zolin, \& Turner, 2009; Vidal, Marle, \& Bocquet, 2011).

Em conformidade com as ideias partilhadas por estes autores, depreende-se que a tomada de decisão é o ponto central do conceito da complexidade. A medida da complexidade 
do projeto para auxiliar na tomada de decisão, bem como a identificação dos múltiplos aspetos da complexidade, de modo a possibilitar apresentação da abordagem multicriterial para avaliação do grau da complexidade do projeto, faz-se do uso do Analytic Hierarchy Process. As medidas multicritério são utilizadas através das escalas e subescalas existentes na estrutura hierárquica para tomada de decisão nos projetos de construção (Vidal et al., 2011).

A complexidade do projeto deverá ser tratada de acordo com o foco da gestão, isto é, o conjunto de decisões e atividades desenvolvidas por indivíduos de modo a atingir os objetivos traçados. A sequência de decisões tomadas determina a trajetória da evolução do projeto, do desempenho da atividade e o resultado final alcançado (Vidal et al., 2011).

Contudo, depreende-se que ao alcançar os resultados finais, poder-se-á encontrar as causas que determinam os desvios orçamentais desfavoráveis, atendendo à comparação dos resultados previstos e os resultados finais (Remington et al., 2009).

Neste âmbito, pretende-se apresentar alguns indicadores de complexidade que possibilitem a mensuração das causas que determinam os desvios orçamentais desfavoráveis nas empresas Portuguesas de construção através da elevada interdependência entre diferentes equipamentos, interdependência dos objetivos, dependência entre níveis hierárquicos, existência de diversos especialistas e profissionais para execução do projeto e existência de riscos e incertezas (Baccarini, 1996; Brockmann \& Girmscheid, 2007; Remington et al., 2009; Van der Stede, 2001). Todavia, procede-se com a abordagem do método de financiamento como causas dos desvios orçamentais desfavoráveis nos projetos das empresas de construção portuguesa.

\subsection{Fator financiamento do projeto como causa dos desvios orçamentais desfavoráveis}

Com a competitividade, as organizações procuram de alguma forma encontrar os melhores instrumentos de financiamento para os seus projetos de construção. Ora como é sabido, existem atualmente vários produtos bancários que permitem financiar projetos de construção, mesmo na sua fase de elaboração e aprovação do projeto (Chirkunova, Kireeva, Kornilova, \& Pschenichnikova, 2016).

O setor bancário e o mercado de valores mobiliários dispõem de ativos financeiros suficientes que poderão ser utilizados para financiar os projetos de construção através de vários instrumentos, tais como empréstimos bancários, empréstimos obrigacionistas, emissão de novas ações, entre outros. No processo de implementação de qualquer projeto, um dos principais componente do sucesso é a escolha correta do instrumento de financiamento (Chirkunova et al., 2016).

De acordo com Brealey et al. (2018) o financiamento é a realização de um empréstimo a credores com a promessa de pagar a dívida acrescida com os respetivos juros. Os autores salientaram que o financiamento poderá ser efetuado com os acionistas, neste caso através do aumento do capital próprio.

Contudo, pretende-se apresentar alguns indicadores de financiamento que proporcionem a mensuração das causas que determinem os desvios orçamentais desfavoráveis, considerando as formas utilizadas para a obtenção dos financiamentos, nomeadamente capital próprio, taxas de juro, créditos aos fornecedores, empréstimo factoring, etc. (Brealey et al., 2018; Brigham \& Ehrhardt, 2013; Chirkunova et al., 2016; Gitman, Juchau, \& Flanagan, 2010; Hackethal \& Schmidt, 2004). É de salientar que o financiamento interno apresenta uma enorme vantagem de custo sobre o financiamento externo, no que diz respeito o custo do capital. A seguir será apresentado o modelo de estudo adotado. 
Mendes, C. C., Couto, J. P., Ferreira, J. V. \& Mendes, E.

Causas dos desvios orçamentais desfavoráveis e os seus constructos: o caso dos projetos das empresas de construção portuguesas

\subsection{Modelo conceptual de estudo}

Na Figura 1 apresenta-se o modelo de estudo e respetivas hipóteses que analisam a influência das causas dos desvios orçamentais desfavoráveis, considerando a utilização do software SPSS (Statistical Package for Social Sciences), versão 25 e AMOS Graphics 25.

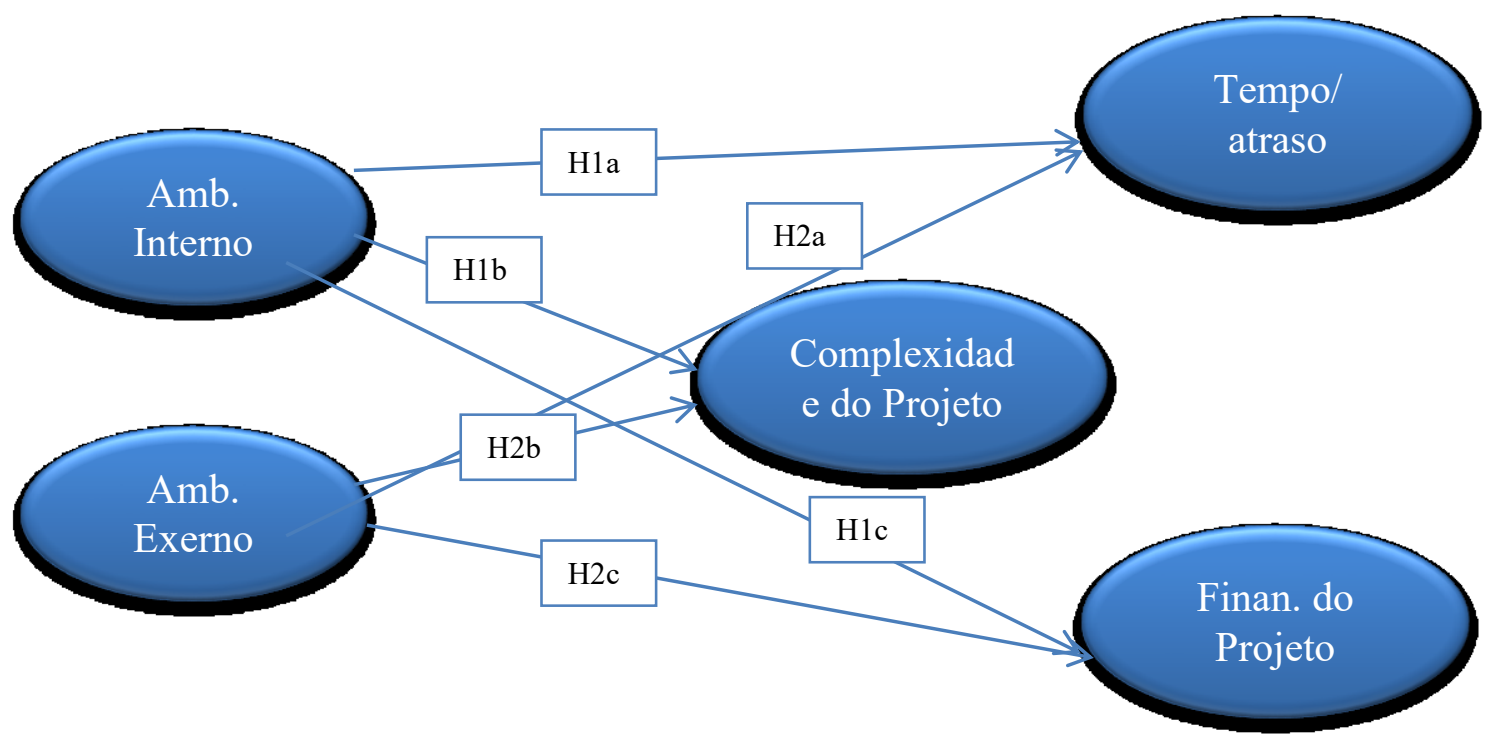

Figura 1. Modelo de estudo

As mensurações dos constructos apresentados no modelo, foram estruturadas em estudos previamente realizados por diversos autores nomeadamente (Mendes, 2019).

As afirmações teóricas que relacionam estes constructos com as causas dos desvios orçamentais desfavoráveis nos projetos das empresas de construção encontram-se descritas no Quadro 1.

\begin{tabular}{|c|c|c|}
\hline $\begin{array}{c}\text { Construct } \\
\text { os/Variáv } \\
\text { eis }\end{array}$ & Cód. & Descrição \\
\hline \multirow{4}{*}{$\begin{array}{c}\text { Fator } \\
\text { Ambiental } \\
\text { Interno }\end{array}$} & FAI1 & $\begin{array}{l}\text { Inexistência de um ambiente organizacional capaz de desenvolver espírito de } \\
\text { equipa }\end{array}$ \\
\hline & FAI2 & $\begin{array}{l}\text { Alterações frequentes no sistema de gestão que condicionam a atividade da } \\
\text { empresa }\end{array}$ \\
\hline & FAI3 & Falta de líderes com capacidade de conduzir as equipas de trabalho \\
\hline & FAI4 & Conflito entre as unidades funcionais na organização da equipa de trabalho \\
\hline \multirow{5}{*}{$\begin{array}{c}\text { Fator } \\
\text { Ambiental } \\
\text { Externo }\end{array}$} & FAE5 & Condições climatéricas \\
\hline & FAE6 & Oscilação da procura dos serviços prestados pelas empresas de construção \\
\hline & FAE7 & Alterações na estrutura do mercado onde a empresa atua \\
\hline & FAE8 & Alteração na atitude dos clientes para com a empresa \\
\hline & FAE9 & $\begin{array}{l}\text { Alterações imprevisíveis nos aspetos sociais, políticos e culturais no meio onde a } \\
\text { empresa atua }\end{array}$ \\
\hline \multirow{3}{*}{$\begin{array}{c}\text { Fator } \\
\text { Tempo/At } \\
\text { raso }\end{array}$} & FT/A1 & Incumprimento dos detalhes do contrato de prestação de serviço \\
\hline & FT/A2 & Escassez de material necessário \\
\hline & FT/A3 & Fraca monitorização e controlo dos materiais em obra \\
\hline
\end{tabular}


Mendes, C. C., Couto, J. P., Ferreira, J. V. \& Mendes, E.

Causas dos desvios orçamentais desfavoráveis e os seus constructos: o caso dos projetos das empresas de construção portuguesas

\begin{tabular}{|c|c|c|}
\hline & FT/A4 & Baixa eficiência e produtividade em resultado de uma comunicação ineficiente \\
\hline & FT/A5 & Escassez de mão-de-obra qualificada \\
\hline & FT/A6 & Escassez de profissionais técnicos na organização e preparação da obra \\
\hline & FT/A7 & Greves no decorrer da obra \\
\hline & FT/A8 & Estudo técnico inadequado durante a fase do concurso \\
\hline & FT/A9 & Dificuldade de comunicação entre os intervenientes \\
\hline & $\begin{array}{c}\text { FT/A1 } \\
0\end{array}$ & Absentismo \\
\hline & $\begin{array}{c}\text { FT/A1 } \\
1\end{array}$ & Inexperiência das equipas designadas para a obra \\
\hline \multirow{7}{*}{$\begin{array}{c}\text { Fator } \\
\text { Complexi } \\
\text { dade dos } \\
\text { Negócios }\end{array}$} & FC1 & Exigências sucessivamente maiores por parte dos clientes \\
\hline & FC2 & Elevada interdependência entre diferentes equipamentos envolvidos \\
\hline & FC3 & Dependência entre níveis hierárquicos na tomada de decisão \\
\hline & FC4 & Interdependência dos objetivos \\
\hline & FC5 & Existência de uma estratégia pouco clara \\
\hline & FC6 & Exigência de diversos especialistas e profissionais para execução dos projetos \\
\hline & FC7 & $\begin{array}{l}\text { Existência de riscos e incertezas próprias das características do projeto que } \\
\text { podem causar impactos desfavoráveis no orçamento }\end{array}$ \\
\hline \multirow{13}{*}{$\begin{array}{c}\text { Fator } \\
\text { Financia } \\
\text { mento }\end{array}$} & \multicolumn{2}{|r|}{ Instrumentos de financiamento a médio e longo prazo } \\
\hline & FFLP1 & Retenção dos resultados ou injeção do capital fresco \\
\hline & FFLP2 & Terem utilizado empréstimos bancários num contexto de subida de taxas de juros \\
\hline & FFLP3 & Não terem utilizado incentivos comunitários \\
\hline & FFLP4 & Terem utilizado recursos financeiros erradamente \\
\hline & \multicolumn{2}{|r|}{ Instrumentos de financiamento a curto prazo } \\
\hline & FFCP5 & Terem utilizado excesso financiamento bancário \\
\hline & FFCP6 & Terem utilizado excesso de créditos de fornecedores \\
\hline & FFCP7 & Terem utilizado em excesso as livranças \\
\hline & FFCP8 & Terem utilizado em excesso desconto de letras \\
\hline & FFCP9 & Problemas e atrasos com financiamentos solicitados \\
\hline & $\begin{array}{c}\text { FFCP1 } \\
0\end{array}$ & Falta de contrato factoring \\
\hline & $\begin{array}{c}\text { FFCP1 } \\
1\end{array}$ & Terem recorrido o financiamento para cobrir alterações nos preços de materiais \\
\hline
\end{tabular}

Quadro 1. Causas dos desvios orçamentais desfavoráveis

No Quadro 2 apresenta-se as hipóteses do estudo, de modo a facilitar uma melhor compreensão dos resultados previamente apurados. 
Mendes, C. C., Couto, J. P., Ferreira, J. V. \& Mendes, E.

Causas dos desvios orçamentais desfavoráveis e os seus constructos: o caso dos projetos das empresas de construção portuguesas

\begin{tabular}{|c|l|}
\hline \multicolumn{2}{|c|}{ Hipótese } \\
\hline H1a & $\begin{array}{l}\text { As causas dos desvios orçamentais desfavoráveis devido ao ambiente interno do projeto } \\
\text { influência positivamente o fator tempo/atraso; }\end{array}$ \\
\hline H1b & $\begin{array}{l}\text { As causas dos desvios orçamentais desfavoráveis devido ao ambiente interno do projeto afeta } \\
\text { positivamente o fator complexidade do projeto; }\end{array}$ \\
\hline H1c & $\begin{array}{l}\text { As causas dos desvios orçamentais desfavoráveis devido ao ambiente interno do projeto } \\
\text { influência positivamente o financiamento do projeto; }\end{array}$ \\
\hline H2a & $\begin{array}{l}\text { As causas dos desvios orçamentais desfavoráveis devido ao ambiente externo do projeto afeta } \\
\text { positivamente o fator tempo/atraso do projeto; }\end{array}$ \\
\hline H2b & $\begin{array}{l}\text { As causas dos desvios orçamentais desfavoráveis devido ao ambiente externo do projeto } \\
\text { influência positivamente o fator complexidade do projeto; }\end{array}$ \\
\hline H2c & $\begin{array}{l}\text { As causas dos desvios orçamentais desfavoráveis devido ao ambiente externo do projeto afeta } \\
\text { positivamente o financiamento do projeto; }\end{array}$ \\
\hline
\end{tabular}

Quadro 2. Hipóteses do estudo

\section{METODOLOGIA DE INVESTIGAÇÃO}

\subsection{Amostra e recolha de dados}

Para Zikmund, Babin, Carr \& Griffin (2002), amostra é um processo que o investigador utiliza para selecionar um certo número de população ou alvo que proporciona tirar as conclusões da investigação.

No universo de 925 empresas de construção, obteve-se uma amostra de 218 observações dos questionários aplicados. Os dados foram recolhidos através dos diretores de produção, gestores, diretores financeiros e CEO/Administradores das empresas de construção portuguesa. A escala utilizada no questionário é do tipo Likert de 1 a 7 (ou seja de descordo em absoluto até concordo em absoluto). Os questionários foram maioritariamente recolhidos através da plataforma web "LimeSurvey".

De seguida, apresenta-se abordagem sobre o modelo de equação estrutural adotado neste estudo.

\subsection{Modelo de equações estruturais}

O estudo adota método de investigação quantitativa, utilizando o modelo de equação estrutural (MEE) através da técnica de análise fatorial exploratória e confirmatório, de modo a permitir ao investigador explorar as principais dimensões para gerar uma teoria ou modelo, a partir de um conjunto de variáveis latentes relativamente grande que muitas vezes são representados por um conjunto de itens (Williams, Onsman, \& Brown, 2010), tendo em conta, a analise das causas dos desvios orçamentais desfavoráveis dos projetos das empresas de construção portuguesa e em seguida confirmar a viabilidade do modelo proposto na Figura 1. O MEE procura instituir as relações entre variáveis latentes e os constructos observáveis, bem 
Mendes, C. C., Couto, J. P., Ferreira, J. V. \& Mendes, E.

Causas dos desvios orçamentais desfavoráveis e os seus constructos: o caso dos projetos das empresas de construção portuguesas

como os relacionamentos entre as variáveis latentes exógenas e as endógenas, atendendo a combinação da análise fatorial confirmatória e da análise de caminhos (path analysis). Para melhor compreensão da análise do modelo, apresenta-se no Quadro 3 as referências de alguns índices de medidas de ajustamento do modelo.

\begin{tabular}{|c|c|c|}
\hline Índice & Descrição & Valores de referência \\
\hline $\begin{array}{l}\text { Goodness-of- } \\
\text { Fit Index } \\
\text { (GFI) }\end{array}$ & $\boldsymbol{G F I}=1-\frac{\operatorname{tr}\left(\widehat{\Sigma^{-1}} S-1\right)^{2}}{\operatorname{tr}\left(\Sigma^{-1} S\right)^{2}}$ & $\begin{array}{l}\text { Valores superiores a 0,90 ajustamento } \\
\text { bom (Hooper et al., 2008; Schumacker } \\
\text { e Lomax, 2010) } \\
\text { Valores superiores a 0,95 ajustamento } \\
\text { muito bom (Shevlin \& Miles, 1998) }\end{array}$ \\
\hline $\begin{array}{l}\text { Adjusted } \\
\text { Goodness-of- } \\
\text { Fit Index } \\
\text { (AGFI) }\end{array}$ & $\boldsymbol{A G F I}=1-\frac{K(K+1)}{2 g l}(1-G F I)$ & $\begin{array}{l}\text { Valores superiores a 0,90 ajustamento } \\
\text { bom (Lomax \& Schumacker, 2010) }\end{array}$ \\
\hline $\begin{array}{l}\text { Root Mean } \\
\text { Square Error } \\
\text { of } \\
\text { Approximation } \\
\text { (RMSEA) }\end{array}$ & RMSEA $=\sqrt{\frac{x^{2}-g l}{\operatorname{gl}(N-1)}}$ & $\begin{array}{l}\text { Valores incluídos entre } 0,05 \text { e } 0,08 \\
\text { (Schumacker e Lomax, 2010) } \\
\text { Valores inferiores a 0,06 (Hu \& } \\
\text { Bentler, 1999) } \\
\text { Valores inferiores a } 0,07 \text { (Steiger, } \\
\text { 2007) } \\
\text { P-value (PCLOSE) superior a 0,5 } \\
\text { (Kline, 2011) }\end{array}$ \\
\hline $\begin{array}{l}\text { Normed Fit } \\
\text { Index (NFI) }\end{array}$ & $\begin{array}{l}\qquad \boldsymbol{N F I}=1-\frac{x^{2}}{x^{2} n u l l} \\
x^{2} \text { null representa o modelo básico ou } \\
\text { nulo }\end{array}$ & $\begin{array}{l}\text { Valores superiores a 0,90 ajustamento } \\
\text { bom (Byrne, 2010; Lomax \& } \\
\text { Schumacker, 2010) } \\
\text { Valores superiores a 0,95 ajustamento } \\
\text { muito bom (Hooper et al., 2008) }\end{array}$ \\
\hline $\begin{array}{l}\text { Tucker-Lewis } \\
\text { Index (TLI) }\end{array}$ & $\boldsymbol{T L} \boldsymbol{I}=\frac{\frac{x^{2} n u l l}{g \ln u l l}-\frac{x^{2}}{g l}}{\frac{x^{2} n u l l}{g \ln u l l}-1}$ & $\begin{array}{l}\text { Valores superiores a 0,90 ajustamento } \\
\text { bom (Schumacker e Lomax, 2010) } \\
\text { Valores superiores a 0,95 ajustamento } \\
\text { muito bom (Hooper et al., 2008; Hu e } \\
\text { Bentler, 1999) }\end{array}$ \\
\hline $\begin{array}{l}\text { Comparative } \\
\text { Fit Index }(\mathrm{CFI})\end{array}$ & $\boldsymbol{C F} \boldsymbol{I}=\frac{x^{2}-g l}{x^{2} \text { null }-g \ln u l l}$ & $\begin{array}{l}\text { Valor superior a } 0,90 \text { ajustamento bom } \\
\text { (Byrne, 2010) } \\
\text { Valor superior a } 0,95 \text { ajustamento } \\
\text { muito bom (Hu e Bentler, 1999) }\end{array}$ \\
\hline $\begin{array}{l}\text { Parcimony } \\
\text { Normed Fit } \\
\text { Index (PNFI) }\end{array}$ & $P N F I=N F I \frac{g l}{g \ln u l l}$ & $\begin{array}{l}\text { Valor superior a } 0,6 \text { ajustamento bom } \\
\text { (Blunch, 2008). }\end{array}$ \\
\hline $\begin{array}{l}\text { Parcimony } \\
\text { Goodness-of- } \\
\text { Fit } \quad \text { Index } \\
\text { (PGFI) }\end{array}$ & $P G F I=G F I \frac{g l}{g l n u l l}$ & $\begin{array}{l}\text { Valor superior a } 0,6 \text { ajustamento bom } \\
\text { (Blunch, 2008) }\end{array}$ \\
\hline
\end{tabular}

Quadro 3. Índices de medida de ajustamento do modelo

\section{ANÁLISE E DISCUSSÃO DOS RESULTADOS}

A caraterização da amostra relativamente ao cargo que o respondente ocupa na empresa, mostra que a taxa de resposta dos gestores é cerca de $28,4 \%$, seguida pelo diretor de produção com $26,6 \%$, sendo o valor mais baixo respeitante ao Diretor financeiro que possui $12,4 \%$. Estes resultados encontram-se indicados na Tabela 1. 
Mendes, C. C., Couto, J. P., Ferreira, J. V. \& Mendes, E.

Causas dos desvios orçamentais desfavoráveis e os seus constructos: o caso dos projetos das empresas de construção portuguesas

Tabela 1. Cargos que os respondes ocupam na empresa

\begin{tabular}{ccc|c|c|c}
\hline & & Frequência & Percentagem & $\begin{array}{c}\text { Percentagem } \\
\text { válida }\end{array}$ & $\begin{array}{c}\text { Percentagem } \\
\text { acumulativa }\end{array}$ \\
\hline \multirow{2}{*}{ Válido } & Gestor & 62 & 28,4 & 34,1 & 34,1 \\
\cline { 2 - 6 } & Diretor Financeiro & 27 & 12,4 & 14,8 & 48,9 \\
\cline { 2 - 6 } & Diretor de Produção & 58 & 26,6 & 31,9 & 80,8 \\
\cline { 2 - 6 } & CEO/Administrador & 35 & 16,1 & 19,2 & 100,0 \\
\cline { 2 - 6 } & Total & 182 & 83,5 & 100,0 & \\
\hline \multirow{2}{*}{ Omisso } & Não Responderam & 36 & 16,5 & & \\
\hline \multirow{2}{*}{} & Total & 218 & 100,0 & & \\
\hline
\end{tabular}

Para determinar a adequação da análise fatorial exploratória, procurou-se avaliar a qualidade das correlações entre as variáveis através de medida de Kaiser.Meyer.Olkin (KMO) e do teste de esfericidade de Bartlett conforme apresentado na Tabela 2.

Todavia, a análise fatorial exploratória utilizada para avaliar a estrutura fatorial das variáveis aponta cinco fatores "ambiente interno, ambiente externo, tempo/atraso, complexidade do projeto e financiamento do projeto" exibindo uma boa correlação entre as mesmas variáveis. Os cinco fatores explicam $68,63 \%$ da variância total acima do limite inferior aceitável 0,60 (Nakip, 2003). O teste KaiserMeyer-Olkin e de esfericidade de Bartlett foram considerados significativos com os níveis de significâncias de 0,919 e 0,000. De salientar que, os resultados apresentado nos testes de KaiserMeyer-Olkin e de esfericidade de Bartlett, mostra que ambos os testes apresentam adequação dos dados de modo a possibilitar a continuidade de análise fatorial exploratória (Hair et al., 2014). Os cinco constructos foram executados através de análise fatorial exploratória utilizando o método de componentes principais com rotação varimax conforme a tabela 2. Também, ao verificar o alfa de Cronbach, percebe-se que, os valores são superiores a 0,72 revelando que os constructos têm uma boa consistência interna. 
Mendes, C. C., Couto, J. P., Ferreira, J. V. \& Mendes, E.

Causas dos desvios orçamentais desfavoráveis e os seus constructos: o caso dos projetos das empresas de construção portuguesas

Tabela 2. Resultado da análise fatorial exploratória

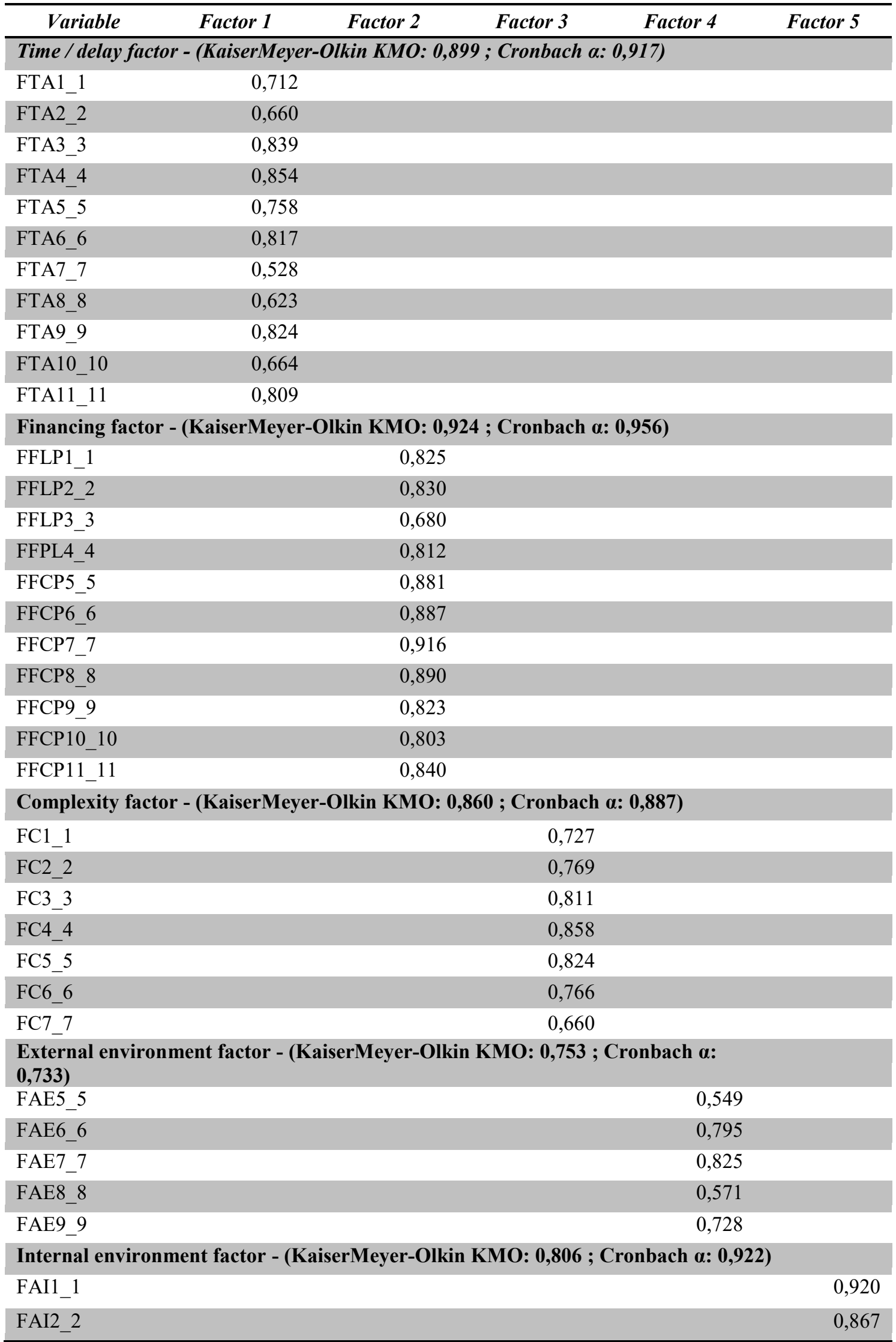


Mendes, C. C., Couto, J. P., Ferreira, J. V. \& Mendes, E.

Causas dos desvios orçamentais desfavoráveis e os seus constructos: o caso dos projetos das empresas de construção portuguesas

\begin{tabular}{lr}
\hline FAI3_3 & 0,914 \\
FAI4_4 & 0,901 \\
\hline
\end{tabular}

Assim, apresenta-se e discutem-se na seção a seguir, os principais resultados da análise fatorial confirmatória. Neste âmbito, procura-se avaliar na Figura 2 a qualidade da relação existente entre variáveis observadas e os respetivos constructos. Em referência a Tabela 2, percebe-se que os resultados da primeira figura do modelo de mensuração apresentam um fraco ajustamento do modelo aos dados da amostra, conforme apresentado nos resultados dos índices da qualidade de ajustamento: $x^{2}=2007,377 ; \mathrm{gl}=655 ; \mathrm{p}$-value $=0,000$; $x^{2} / \mathrm{gl}=3,064 ; \mathrm{RMSA}=0,098 ; \mathrm{PCLOSE}=0,000 ; \mathrm{TLI}=0,786 ; \mathrm{CFI}=0,801 ; \mathrm{PCFI}=0,746 ;$ $\mathrm{NFI}=0,732$.

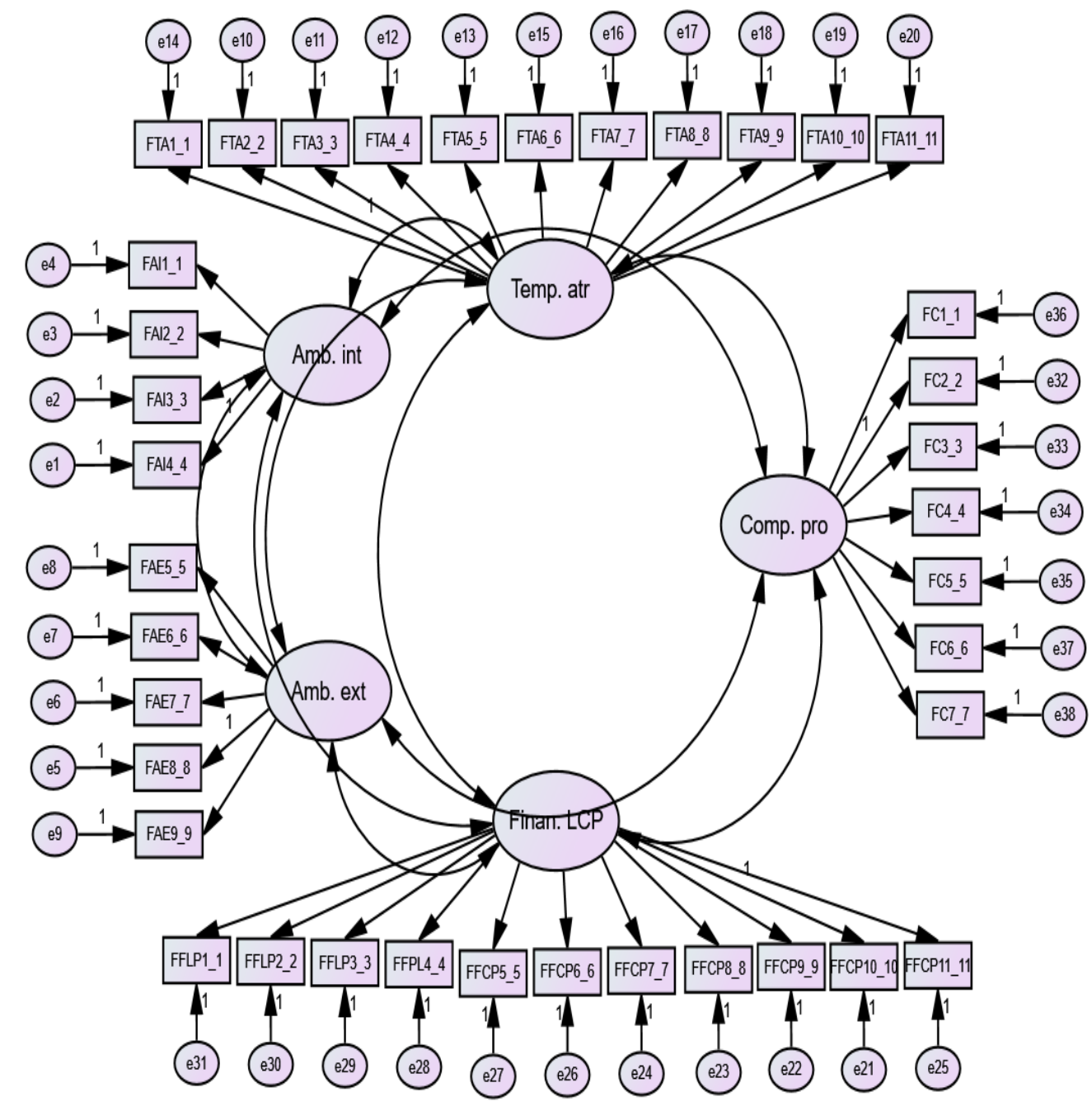

Figura 2. Modelo de medida: Causas dos desvios orçamentais desfavoráveis

Tendo em conta os resultados apresentados na Tabela 2 e na Figura 2, depreende-se que, os resultados das análises apresentam uma fraca qualidade de ajustamento do modelo, de modo que, é imprescindível a utilização da análise de modification indices e aos resíduos estandardizados para possibilitar a melhoria no ajustamento dos dados (Hair et al., 2014; Lomax \& Schumacker, 2010). 
Neste âmbito, foram selecionados e excluídos os indicadores FFCP7, FFLP2 e FFLP3 por apresentarem o índice de modificação entre o seu termo de erro de 94,130; 90,498 e 42,248 e o termo de erro dos indicadores FFCP8, FFLP1 e FFLP1.

De salientar que, os indicadores FAI2, FC7, FTA7, FTA4, FC2 e FFCP5 foram removidos devido aos elevados índices de modificação entre o seu termo de erro de 36,880; 32,$612 ; 31,279 ; 31,921 ; 28,066$ e 26,001 com o termo de erro dos indicadores FAI1, FC6, FTA10, FTA3, FC1 e FFCP6. Na mesma linha de análise, os indicadores FTA5, FFPL4 e FC3 foram excluídos devido aos elevados índices de modificação entre o seu termo de erro de 25,017; 23,600 e 16,974 com o termo de erro dos indicardes FTA6, FFLP1 e FC1.

Por último, foi excluído o indicador FTA8 devido aos elevados crossloadings com o Ambiente interno do projeto, apresentando o índice de modificação de 19,132.

Contudo, as modificações dos termos de erros correlacionadas foram evitadas através da exclusão de um dos indicadores em cada par apresentado no paragrafo anterior (Hair et al. 2014).

No que concerne a ré-especificação do modelo inicial e apesar dos testes estatísticos terem revelado a remoção dos indicadores com elevada termos de erros, verifica-se que nos 38 itens apresentados na Tabela 2 só restam 25 itens após o ajustamento do modelo de medida conforme a Tabela 3, mostrando que ficou mais de metade do que as consideradas inicialmente na Tabela 2. Contudo verificou-se que nenhum dos fatores ficou sem classificação, o que significa que cada um deles ocorre, ainda que possa não ser grave e, por isso corrigível, ficando desta forma validado o conjunto dos fatores considerado no estudo (Couto, 2007).

O ajustamento do modelo atual progrediu muito comparando com o modelo inicial, podendo-se provar através dos resultados dos seguintes índices: $x^{2}=513,335 ; \mathrm{gl}=265 ; \mathrm{p}$ value $=0,000 ; x^{2} / \mathrm{gl}=1,937 ; \mathrm{RMSA}=0,066 ; \mathrm{PCLOSE}=0,000 ; \mathrm{IFI}=0,928 ; \mathrm{TLI}=0,918$; $\mathrm{CFI}=0,928 ; \mathrm{PCFI}=0,819 ; \mathrm{NFI}=0,863$. Com os resultados apresentados, verifica-se a consistência interna do modelo. No entanto, segue o modelo ajustado na Figura 3.

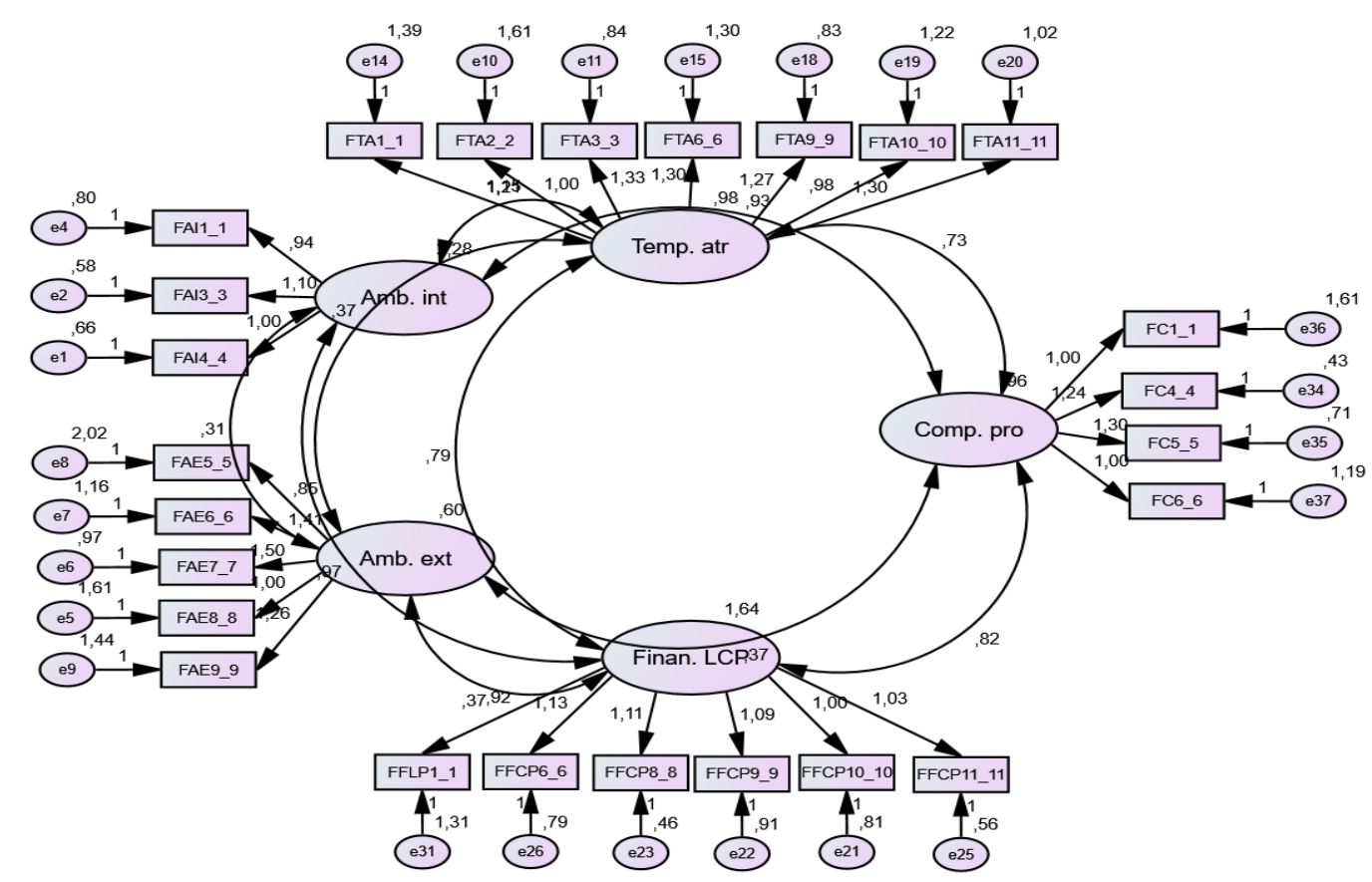

Figura 3. Modelo de medida ajustado: Causas dos desvios orçamentais desfavoráveis 
Sendo assim, é imprescindível analisar a validade convergente do modelo e a fiabilidade dos constructos através da fiabilidade compósita (FC), da variância média extraída (VME) e do alfa de Cronbach $(\alpha)$ (Fornell \& Larcker, 1981). Nesta periferia, depreende-se que os resultados apresentados na Tabela 3 no qual se verifica que o Alfa de Cronbach $(\alpha)$ indica a fiabilidade do constructo com valores superiores a 0,729. A fiabilidade compósita (FC) foi representada com valores superiores a 0,876 , confirmando a consistência interna. Por último, a variância média extraída (VME) surge com valores acima de 0,500 recomendando a validade convergente adequada (Hair et al., 2014).

Tabela 3. Resultados da análise fatorial confirmatória do modelo de medida ajustado

\begin{tabular}{|c|c|c|c|c|}
\hline Variables & Factor loading & $\alpha$ & FC & VME \\
\hline \multicolumn{2}{|c|}{ Internal environment factor } & \multirow{4}{*}{0,909} & \multirow{4}{*}{0,950} & \multirow{4}{*}{0,772} \\
\hline FAI4 & 0,881 & & & \\
\hline FAI3 & 0,909 & & & \\
\hline FAI1 & 0,844 & & & \\
\hline \multicolumn{2}{|c|}{ External environment factor } & \multirow{6}{*}{0,733} & \multirow{6}{*}{0,908} & \multirow{6}{*}{$\mathbf{0 , 5 3 9}$} \\
\hline FAE8 & 0,681 & & & \\
\hline FAE7 & 0,762 & & & \\
\hline FAE5 & 0,862 & & & \\
\hline FAE6 & 0,712 & & & \\
\hline FAE9 & 0,631 & & & \\
\hline \multicolumn{2}{|l|}{ Time / delay factor } & \multirow{8}{*}{$\mathbf{0 , 8 8 9}$} & \multirow{8}{*}{0,934} & \multirow{8}{*}{0,698} \\
\hline FTA2 & 0,915 & & & \\
\hline FTA3 & 0,821 & & & \\
\hline FTA1 & 0,895 & & & \\
\hline FTA9 & 0,810 & & & \\
\hline FTA10 & 0,860 & & & \\
\hline FTA11 & 0,786 & & & \\
\hline FTA6 & 0,749 & & & \\
\hline \multicolumn{2}{|l|}{ Complexity factor } & \multirow{5}{*}{$\mathbf{0 , 8 3 1}$} & \multirow{5}{*}{0,889} & \multirow{5}{*}{$\mathbf{0 , 5 8 6}$} \\
\hline FC5 & 0,835 & & & \\
\hline $\mathrm{FC} 4$ & 0,880 & & & \\
\hline $\mathrm{FC} 1$ & 0,655 & & & \\
\hline FC6 & 0,667 & & & \\
\hline \multicolumn{2}{|l|}{ Financing factor } & \multirow{7}{*}{$\mathbf{0 , 9 3 0}$} & \multirow{7}{*}{0,961} & \multirow{7}{*}{0,694} \\
\hline FFCP11 & 0,871 & & & \\
\hline FFLP1 & 0,718 & & & \\
\hline FFCP6 & 0,851 & & & \\
\hline FFCP8 & 0,901 & & & \\
\hline FFCP9 & 0,827 & & & \\
\hline FFCP10 & 0,819 & & & \\
\hline
\end{tabular}


Ao averiguar a Tabela 3 depreende-se que o modelo ajustado não apresenta problemas de validade discriminante, ou seja, os constructos apresentados no estudo são diferentes entre si e o VME é maior que o quadrado da correlação entre os mesmos constructos (Fornell e Larcker, 1981). Assim, segue-se a matriz de correlação dos constructos.

Tabela 4. Matriz das correlações dos constructos

\begin{tabular}{|c|c|c|c|c|c|c|c|c|}
\hline & 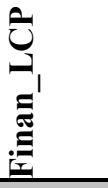 & 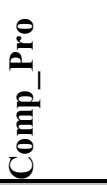 & 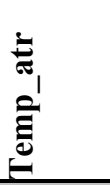 & 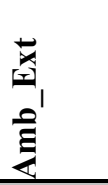 & $\begin{array}{l}\stackrel{\Xi}{\Xi} \\
\stackrel{\Xi}{\Xi}\end{array}$ & $\boldsymbol{\alpha}$ & FC & VME \\
\hline Finan_LCP & 0,694 & & & & & 0,930 & 0,961 & 0,694 \\
\hline Comp_Pro & 0,424 & 0,586 & & & & 0,831 & 0,899 & 0,572 \\
\hline Temp_atr & 0,388 & 0,575 & 0,698 & & & 0,889 & 0,934 & 0,544 \\
\hline Amb_Ext & 0,136 & 0,241 & 0,237 & 0,539 & & 0,733 & 0,908 & 0,539 \\
\hline Amb_Int & 0,393 & 0,252 & 0,661 & 0,071 & 0,772 & 0,909 & 0,950 & 0,772 \\
\hline
\end{tabular}

Obs. os valores na diagonal são a VME. Os valores abaixo da diagonal são o quadrado das correlações entre os constructos.

Contudo, a validação do modelo de medida, seguiu-se para modelo estrutural de modo a possibilitar a confirmação das hipóteses através dos testes realizado, atendendo as relações dos constructos. A seguir, segue a Figura 4 para perceber melhor as relações dos constructos apresentados no estudo.

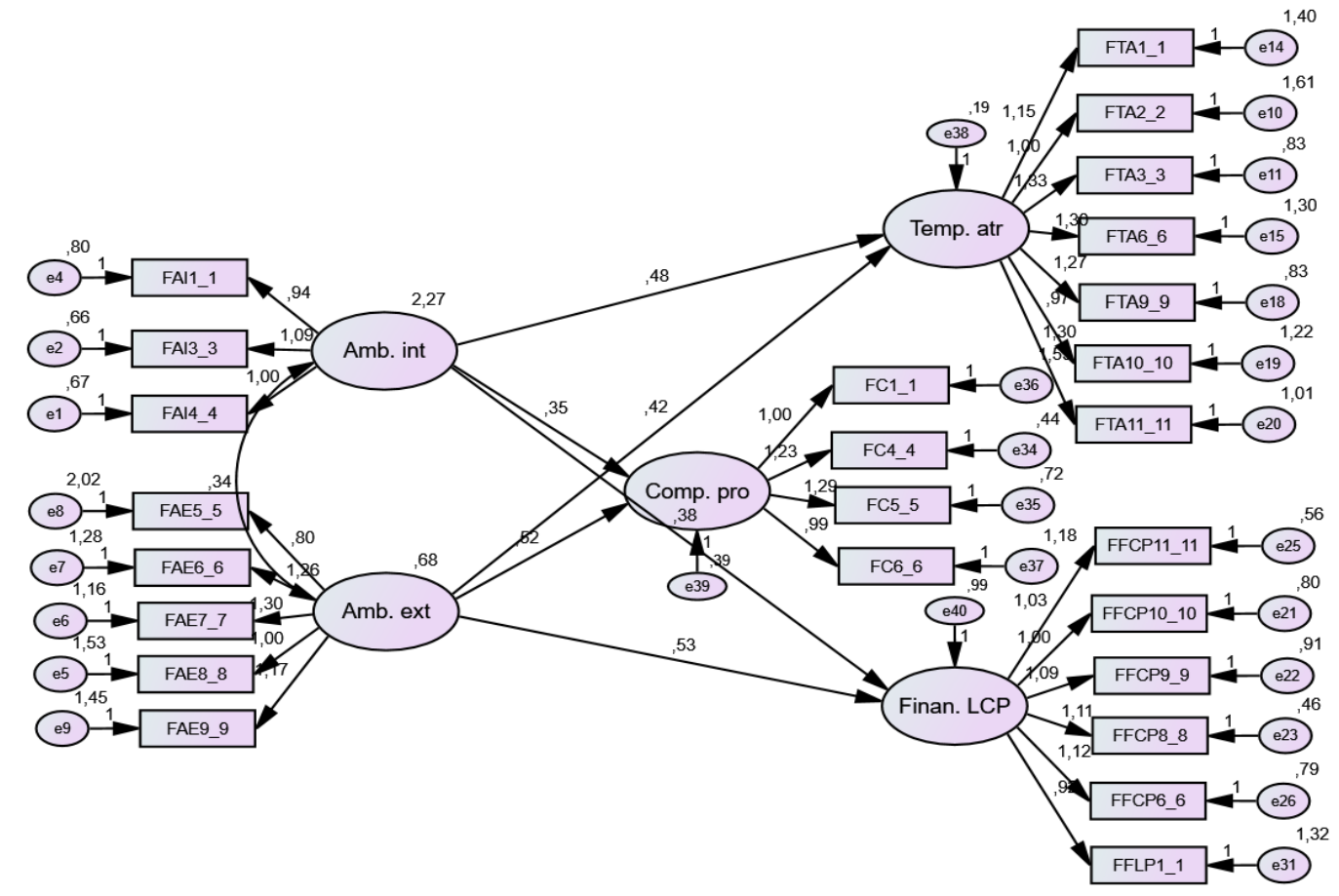

Figura 4. Modelo estrutural: Causas dos desvios orçamentais desfavoráveis

De acordo com os resultados apresentado no Quadro 4, assim como na Figura 4, verifica-se que das 6 hipóteses testadas, todas elas são corroboradas empiricamente com este 
Mendes, C. C., Couto, J. P., Ferreira, J. V. \& Mendes, E.

Causas dos desvios orçamentais desfavoráveis e os seus constructos: o caso dos projetos das empresas de construção portuguesas

estudo. Entretanto, as informações encontradas neste estudo revelam que os seis constructos são as principais causas que determinam os desvios orçamentais desfavoráveis nas empresas de construção portuguesa.

\begin{tabular}{|c|c|c|c|c|c|c|c|}
\hline Hipót & \multicolumn{3}{|c|}{ Relação estrutural } & Factor & $\mathbf{P}$ & $\mathbf{R}^{2}$ & Resultado \\
\hline H1a & Amb_int & $\longrightarrow$ & Temp_atr & 0,483 & $* * *$ & 0,233 & Corroborado \\
\hline $\mathrm{H} 1 \mathrm{~b}$ & Amb_int & $\rightarrow$ & Comp_pro & 0,355 & $* * *$ & 0,126 & Corroborado \\
\hline $\mathrm{H} 1 \mathrm{c}$ & Amb_int & $\rightarrow$ & Finan_LCP & 0,381 & $* * *$ & 0,145 & Corroborado \\
\hline $\mathrm{H} 2 \mathrm{a}$ & Amb_ext & $\longrightarrow$ & Temp_atr & 0,420 & $* * *$ & 0,176 & Corroborado \\
\hline $\mathrm{H} 2 \mathrm{~b}$ & Amb_ext & $\longrightarrow$ & Comp_pro & 0,515 & $* * *$ & 0,265 & Corroborado \\
\hline $\mathrm{H} 2 \mathrm{c}$ & Amb_ext & $\longrightarrow$ & Finan_LCP & 0,530 & $* * *$ & 0,281 & Corroborado \\
\hline
\end{tabular}

Quadro 4. Resultado do modelo estrutural

Ao considerar o ambiente interno do projeto como causa dos desvios orçamentais desfavoráveis, depreende-se que afetam positivamente o fator tempo/atraso, assim como o fator complexidade do projeto e fator financiamento do projeto, corroborando as hipóteses H1a, H1b e H1c. Todavia, estas hipóteses são corroboradas com as hipóteses do estudo de Zidane e Andersen (2018) que observaram o processo de tomada de decisão lento/fraco; procedimentos administrativos internos e a burocracia nas organizações de projetos; escassez de recursos (recursos humanos, máquinas, equipamentos); má comunicação e coordenação entre as partes; alterações de projeto durante a construção; dificuldades financeiras, etc., como os fatores que possibilitem a compreensão das causas que determinam os desvios orçamentais desfavoráveis nos projetos das empresas de construção. Devido ao ambiente externo do projeto, as causas dos desvios orçamentais desfavoráveis afetam positivamente o fator tempo/atraso bem como fator complexidade do projeto e fator financiamento do projeto, corroborando as hipóteses $\mathrm{H} 2 \mathrm{a}, \mathrm{H} 2 \mathrm{~b}$ e H2c. Estas hipóteses foram suportadas nos estudos de Zhao et al. (2019) que tinha apontado as condições do mercado onde a empresa atua, como fatores que contribuíram para explicação das causas dos desvios orçamentais desfavoráveis. As conclusões apresentadas foram confirmadas no estudo "Determinantes dos Desvios orçamentais e o seu impacto na performance das empresas de construção Portuguesa" (Mendes, 2019). No ponto seguinte serão tecidas as considerações e conclusões finais, apresentando igualmente as contribuições e limitações do estudo, bem como as sugestões sobre as investigações futuras.

\section{CONCLUSÕES}

Ao logo do estudo, procurou-se compreender as relações dos constructos que determinam as causas dos desvios orçamentais desfavoráveis nos projetos das empresas de construção Portuguesas.

Todavia, verifica-se na literatura a existência de diversos estudos acerca do incumprimento dos prazos, descontrolo financeiro, derrapagens orçamentais nos projetos de construção (Couto, 2007; Odeck, 2004; Remington et al., 2009; Shehu, Endut, \& Akintoye, 2014; Sridarran et al., 2017). 
Recorrendo à gestão dos inquéritos na plataforma Web "LimeSurvey", obteve-se uma amostra de 218 observações num universo de 925 empresas de construção. As análises foram efetuadas através do software SPSS (Statistical Package for Social Sciences), versão 25 e AMOS Graphics 25.

Os resultados alcançados no estudo mostram a elevada correlação entre os cinco constructos de primeira ordem (ambiente interno, ambiente externo, tempo/atraso, complexidade do projeto e financiamento do projeto), fundamentada pela revisão da literatura, justificam a perceção relativa à identificação dos fatores que determinam as causas dos desvios orçamentais desfavoráveis nos projetos das empresas de construção Portuguesa (Kaming et al., 1997; Odeck, 2004; Couto, 2007; Horngren et al., 2010; Zidane e Andersen, 2018; Zhao et al., 2019).

Contudo, depreende-se que estes resultados corroboram os estudos de Mendes (2019), que considerou as condições climatéricas, a alteração da estrutura do mercado, as alterações dos projetos, a escassez de materiais, as existências de riscos e incertezas do projeto, a interdependência dos objetivos dos projetos, os excessos de créditos aos fornecedores, como as causas dos desvios orçamentais desfavoráveis.

Os resultados encontrados no presente estudo revelam e contribuiriam na identificação dos fatores que determinam as causas dos desvios orçamentais desfavoráveis, de modo a proporcionar aos utilizadores do sistema orçamental um conhecimento acrescido, permitindolhes retirar o maior proveito e acrescentar valor à organização, procurando implementar as correções que visem a mitigação ou eliminação dos possíveis desvios orçamentais desfavoráveis.

As limitações do estudo surgem a partir dos limites estabelecidos para análise do modelo teórico proposto, atendendo as comparações dos resultados com outros estudos. De salientar que, embora o tamanho da amostra utilizado seja apropriado para realização da modelação das equações estruturais, mais dados podem melhorar o ajuste do modelo e a sua validade.

Contudo, os fatores apresentados neste estudo não são os únicos que afetam os determinantes das causas dos desvios orçamentais desfavoráveis nos projetos das empresas de construção, pelo que é necessário realizar investigações futuras para tornar compreensível o assunto. Sugere-se um estudo sobre os desvios orçamentais desfavoráveis de acordo com o ramo de atividade das empresas de construção. Por outro lado, pode-se também fazer um estudo comparativo entre as empresas de construção portuguesa e com as empresas de outros países da Europa.

\section{REFERENCIAS}

Anthony, R. N. (1970). Management accounting principles: RD Irwin.

Arditi, D., Akan, G. T., \& Gurdamar, S. (1985). Cost overruns in public projects. International Journal of Project Management, 3(4), 218-224.

Baccarini, D. (1996). The concept of project complexity - a review. International Journal of Project Management, 14(4), 201-204.

Barki, H., Rivard, S., \& Talbot, J. (1993). Toward an assessment of software development risk. Journal of Management Information Systems, 203-225.

Blunch, N. (2008). Introduction to structural equation modelling using SPSS and AMOS: Sage. 
Brealey, R. A., Myers, S. C., \& Allen, F. (2018). Princípios de Finanças Corporativas-12: AMGH.

Brigham, E., \& Ehrhardt, M. (2013). Financial management: theory \& practice: Cengage Learning.

Brockmann, C., \& Girmscheid, G. (2007). Complexity of megaprojects. Paper presented at the CIB World Building Congress: construction for development: 14-17 May 2007, Cape Town International Convention Centre, South Africa.

Byrne, B. M. (2010). Structural equation modeling with AMOS: Basic concepts, applications, and programming: Routledge.

Cantarelli, C. C., van Wee, B., Molin, E. J., \& Flyvbjerg, B. (2012). Different cost performance: different determinants?: The case of cost overruns in Dutch transport infrastructure projects. Transport Policy, 22, 88-95.

Cheng, Y.-M. (2014). An exploration into cost-influencing factors on construction projects. International Journal of Project Management, 32(5), 850-860.

Chirkunova, E. K., Kireeva, E. E., Kornilova, A. D., \& Pschenichnikova, J. S. (2016). Research of instruments for financing of innovation and investment construction projects. Procedia Engineering, 153, 112-117.

Coulmas, N., \& Law, M. D. (2010). Budgeting in a Chaotic Economic Environment... Factors Leading to Improvement. Journal of Marketing Development and Competitiveness, 5(1), 42-46.

Couto, J. P. (2007). Incumprimento dos prazos na construção. [Dissertação de Doutoramento].

Drucker, P. F. (2009). Management cases: Harper Collins.

Duncan, R. B. (1972). Characteristics of organizational environments and perceived environmental uncertainty. Administrative science quarterly, 313-327.

Flyvbjerg, B., Skamris Holm, M. K., \& Buhl, S. L. (2004). What causes cost overrun in transport infrastructure projects? Transport reviews, 24(1), 3-18.

Fornell, C., \& Larcker, D. F. (1981). Structural equation models with unobservable variables and measurement error: Algebra and statistics. Journal of Marketing research, 382-388.

Gitman, L. J., Juchau, R., \& Flanagan, J. (2010). Principles of managerial finance: Pearson Higher Education AU.

Hackethal, A., \& Schmidt, R. H. (2004). Financing patterns: measurement concepts and empirical results: Working paper series: Finance \& Accounting.

Hair, J. J. F., Black, W. C., Babin, B. J., \& Anderson, R. E. (2014). Multivariate data analysis (Seventh, Pearson new international ed.). Harlow: Pearson Education Limited.

Hansen, S. C., Otley, D. T., \& Van der Stede, W. A. (2003). Practice developments in budgeting: an overview and research perspective. Journal of management accounting research, 15(1), 95-116.

Hartmann, A. (2006). The context of innovation management in construction firms. Construction Management and Economics, 24(6), 567-578. 
Hu, L. t., \& Bentler, P. M. (1999). Cutoff criteria for fit indexes in covariance structure analysis: Conventional criteria versus new alternatives. Structural equation modeling: $a$ multidisciplinary journal, 6(1), 1-55.

Kaming, P. F., Olomolaiye, P. O., Holt, G. D., \& Harris, F. C. (1997). Factors influencing construction time and cost overruns on high-rise projects in Indonesia. Construction Management \& Economics, 15(1), 83-94.

Lee, J.-K. (2008). Cost overrun and cause in Korean social overhead capital projects: Roads, rails, airports, and ports. Journal of Urban Planning and Development, 134(2), 59-62.

Libby, T., \& Lindsay, R. M. (2007). Beyond Budgeting or Better Budgeting? Just how important and useful is budgeting? Survey respondents look at weaknesses that are built in, such as budget gaming, and they ask whether traditional. Strategic Finance, 89(2), 4651.

Lind, H., \& Brunes, F. (2015). Explaining cost overruns in infrastructure projects: a new framework with applications to Sweden. Construction Management and Economics, 33(7), 554-568.

Lomax, R. G., \& Schumacker, R. E. (2010). A beginner's guide to structural equation modeling ( $3^{\mathrm{a}}$ rd ed.): Routledge Academic New York, NY.

Lorain, M.-A. (2010). Should rolling forecasts replace budgets in uncertain environments? Performance Measurement and Management Control: Innovative Concepts and Practices (pp. 177-208): Emerald Group Publishing Limited.

Mao, H., Zhu, B., \& Wang, T. a. (2013). The effect of organizational environment on engineering project cost management: A field study on CREC 2nd Bureau Co. Ltd. Nankai Business Review International, 4(1), 25-48.

Marôco, J. (2010). Análise de equações estruturais: Fundamentos teóricos, software \& aplicações: ReportNumber, Lda.

Mendes, C. C. (2019). Determinantes dos Desvios Orçamentais e o Seu Impacto Na Performance das Empresas de Construção Portuguesas. Faculdade de Economia da Universidade de Coimbra.

Milliken, F. J. (1987). Three types of perceived uncertainty about the environment: State, effect, and response uncertainty. Academy of Management review, 12(1), 133-143.

Nakip, M. (2003). Marketing Researches: Techniques and Applications (with SPSS). Seçkin, Ankara.

Nidumolu, S. (1995). The effect of coordination and uncertainty on software project performance: residual performance risk as an intervening variable. Information Systems Research, 6(3), 191-219.

Odeck, J. (2004). Cost overruns in road construction-what are their sizes and determinants? Transport Policy, 11(1), 43-53.

Remington, K., Zolin, R., \& Turner, R. (2009). A model of project complexity: distinguishing dimensions of complexity from severity. Paper presented at the Proceedings of the 9th International Research Network of Project Management Conference. 
Shehu, Z., Endut, I. R., \& Akintoye, A. (2014). Factors contributing to project time and hence cost overrun in the Malaysian construction industry. Journal of Financial Management of Property and Construction, 19(1), 55-75.

Shevlin, M., \& Miles, J. N. (1998). Effects of sample size, model specification and factor loadings on the GFI in confirmatory factor analysis. Personality and Individual differences, 25(1), 85-90.

Sridarran, P., Keraminiyage, K., \& Herszon, L. (2017). Improving the cost estimates of complex projects in the project-based industries. Built Environment Project and Asset Management, 7(2), 173-184.

Steiger, J. H. (2007). Understanding the limitations of global fit assessment in structural equation modeling. Personality and Individual differences, 42(5), 893-898.

Van der Stede, W. A. (2001). Measuring 'tight budgetary control'. Management Accounting Research, 12(1), 119-137.

Vidal, L.-A., Marle, F., \& Bocquet, J.-C. (2011). Measuring project complexity using the Analytic Hierarchy Process. International Journal of Project Management, 29(6), 718727.

Welsch, G. A. (1957). Budgeting: profit planning and control-5/E: Prentice-Hall.

Williams, B., Onsman, A., \& Brown, T. (2010). Exploratory factor analysis: A five-step guide for novices. Australasian Journal of Paramedicine, 8(3).

Wong, K., \& Vimonsatit, V. (2012). A study of the factors affecting construction time in Western Australia. Scientific Research and Essays, 7(40), 3390-3398.

Worthington, I., \& Britton, C. (2009). The business environment: Pearson Education.

Xiong, B., Skitmore, M., \& Xia, B. (2015). A critical review of structural equation modeling applications in construction research. Automation in construction, 49, 59-70.

Zhao, L., Wang, B., Mbachu, J., \& Liu, Z. (2019). New Zealand Building Project Cost and Its Influential Factors: A Structural Equation Modelling Approach. Advances in Civil Engineering, 2019.

Zidane, Y. J.-T., \& Andersen, B. (2018). The top 10 universal delay factors in construction projects. International Journal of Managing Projects in Business, 11 (3), 650-672. 